\title{
Strategies and results of oncofertility counseling in young breast cancer patients
}

\author{
Joanna Kufel-Grabowska1,2, Piotr Jędrzejczak³, Mikołaj Bartoszkiewicz ${ }^{4}$, Paweł Burchardt \\ Maria Litwiniuk ${ }^{6,7}$
}

\author{
${ }^{1}$ Department of Electroradiology, Poznan University of Medical Sceinces, Poznan, Poland \\ 2Department of Chemotherapy, University Hospital of Lord's Transfiguration, Poznan, Poland \\ ${ }^{3}$ Department of Infertility and Reproductive Endocrinology, Poznan University of Medical Sciences, Poznan, Poland \\ ${ }^{4}$ Department of Immunobiology, Poznan University of Medical Sciences, Poznan, Poland; \\ ${ }^{5}$ Department of Hypertension, Angiology and Internal Medicine, Poznan University of Medical Sciences, Poznan, Poland \\ ${ }^{6}$ Department of Chemotherapy, Greater Poland Cancer Center, Poznan, Poland \\ ${ }^{7}$ Department of Cancer Pathology and Prevention, Poznan University of Medical Sciences, Poznan, Poland
}

Introduction. Breast cancer (BC) is the most common female neoplasm in Poland and worldwide, yet up to $7 \%$ of all cases is diagnosed \&lt; 40 years of age. The increased BC morbidity rate in this age group as well as hope for late maternity need special attention.

Material and methods. The data concerning the number of children and further procreation needs in women $(n=68)$, aged 18-40, diagnosed and treated for early breast cancer at the Greater Poland Cancer Center in 2018-2019, were taken from patients' histories by an oncologist before (neo-)adjuvant systemic therapy.

Results. Out of the 68 females surveyed, aged 18-40 (median age 36), 14 (21\%) were childless at the moment of diagnosis. After being informed about the therapy, prognosis, side effects and oncofertility, 12 patients (18\%) decided to have a consultation with a specialist in reproductive medicine; 5 of them (7\%) already had children. In 2 women (3\%), hormonal stimulation in combination with tamoxifen was used; then, oocytes were collected and cryopreserved. In 19 (28\%), gonadotropine analogues were added to (neo-)adjuvant chemotherapy. In 17 patients (25\%) pathogenic mutations in $B R C A 1 / 2$ genes were found.

Conclusions. Oncofertility counseling in young BC patients should be one of the fundamental elements of complex patient care.

Key words: oncofertility, breast cancer, BRCA1/2 mutation, young women

\section{Introduction}

Breast cancer is the most frequently diagnosed cancer among women in Poland and in the world, but it rarely occurs in young women. Patients under 40 years of age constitute about $7 \%$ of all cases. The prognosis for young women is worse than that of older women mainly due to the more frequent occurrence of unfavorable phenotypes of breast cancer and the presence of numerous genetic disorders in the tumor tissue, which is the reason for the more aggressive course of the disease [1]. In addition, at

\section{How to cite:}

Kufel-Grabowska J, Jędrzejczak P, Bartoszkiewicz M, Burchardt P, Litwiniuk M. Strategies and results of oncofertility counseling in young breast cancer patients NOWOTWORY J Oncol 2021; 71: 263-266.

This article is available in open access under Creative Common Attribution-Non-Commercial-No Derivatives 4.0 International (CC BY-NC-ND 4.0) license, allowing to download articles and share them with others as long as they credit the authors and the publisher, but without permission to change them in any way or use them commercially. 
the time of diagnosis, young women are more often diagnosed with more advanced disease than older women. The therapy of breast cancer, apart from surgery, often requires complementary treatment: chemotherapy, hormone therapy or radiotherapy. Systemic treatment is more likely to temporarily or irreversibly impair fertility than other cancers, which may be due in part to the duration of breast cancer therapy (up to 10 years) [2].

Diagnosis of breast cancer may have devastating effects on a woman and her loved ones, and affects every sphere of their lives. In the case of young women, it often occurs during the period of starting a family and planning offspring. The prospect of having to undergo cancer treatment and the desire to have children should not be mutually exclusive, and any woman in her reproductive years who expresses a desire to have children should have a consultation with a reproductive medicine specialist before starting treatment, preferably immediately after breast cancer diagnosis. If the consultation is delayed, the chances of fertility preservation after treatment are reduced [2].

The oncologist's role is to present the patient with a treatment plan, and to inform her about the possible effects of therapy, including the potential impact on ovarian dysfunction. The stress associated with a cancer diagnosis causes woman to postpone procreation plans, and instead focus on the cancer therapy. For most patients with early breast cancer, postponing therapy by 3-4 weeks does not affect prognosis. During this time, patients can take care of fertility preservation and enjoy motherhood after treatment. A significant proportion of young breast cancer patients are diagnosed with a mutation in their genes that increases the risk of breast and ovarian cancer (BRCA1/2), and the only treatment to reduce the risk of developing subsequent cancers is surgery: bilateral mastectomy and ovariectomy [3]. Carrying pathogenic mutations in BRCA1/2 genes by the patient can impact decisions to have offspring after breast cancer therapy and before ovarian removal.

\section{Objective}

The aim of this paper was to assess the interest of young patients with early breast cancer in fertility preservation techniques, treated in the Chemotherapy Outpatient Clinic of the Greater Poland Cancer Center, Poznan, Poland, in consultation with a reproductive medicine specialist. An additional aim was to draw physicians' attention to the problem of infertility accompanying cancer therapy.

\section{Material and methods}

It was a retrospective study. Patients with early breast cancer aged 18-40 years who were treated in the Chemotherapy Outpatient Clinic of the Greater Poland Cancer Center at Poznan, Poland during 2018-2019 and informed about the possibility of fertility preservation techniques were covered with the study. Medical data were obtained from medical history regarding age, offspring, desire to have children in the future, consultation with a reproductive medicine specialist, and fertility preservation techniques used, as well as carrying pathogenic mutations in genes that increase breast cancer risk. Fertility preservation techniques included ovarian stimulation, oocyte collection and freezing, as well as the inclusion of gonadotropin-releasing hormone analoges during systemic treatment.

\section{Statistic}

The IBM SPSS Statistics 26 program was used for the analysis. The significance level was adopted as 0.05. The Shapiro-Wilk test was used to test the normality of data distribution. The nonparametric Mann-Whitney $U$ test was used to compare the data distributions (due to the lack of normal data distribution). The chi-square test and z-test were used to investigate correlations.

\section{Ethics}

An oncologist and gynecologist's analysis of patient records does not require the opinion of a bioethical committee.

\section{Results}

66 patients with early breast cancer who underwent treatment in the Chemotherapy Outpatient Clinic of the Greater Poland Cancer Center at Poznan, Poland during 2018-2019 were included in the analysis. The median age of patients was 36 years ( $26-40$ years). All patients were informed by the treating physician about the potential effects of systemic treatment and the possibility of fertility preservation techniques prior to systemic treatment. Those who received consultation with a reproductive medicine specialist were characterized by their younger age (33.5 vs. 36.5 years).

At the time of treatment planning for early breast cancer, 14 patients (20.6\%) had no children. After discussion with the oncologist about prognosis, treatment plan, and the possibility of complications, including infertility, 12 patients (17.6\%) decided to have a consultation with a reproductive medicine specialist; among those without children ( $n=14$ patients), half ( $n=7$ patients, 50\%) had a consultation with a specialist, whereas among those with at least one child, less than 10\% ( $n=5$ of a group of $n=54$ patients; $9.3 \%$ ) decided to have such a consultation. 19 patients (27.9\%) were treated with gonadotropin-releasing hormone $(\mathrm{GnRH})$ analoges, while 2 patients (2.9\%) underwent hormonal stimulation combined with tamoxifen, followed by oocyte collection and freezing (tab. I).

Table I. Applied fertility preservation techniques

\begin{tabular}{|lc|}
\hline Fertility preservation techniques & No. of patients (\%) \\
\hline oocyte freezing & $n=2(2.9)$ \\
\hline use of GnRH & $n=19(27.9)$
\end{tabular}


Table II. Test results found in medical history

Type of test
test for founder mutations in BRCA1 gene
(c.5266dupC, c.4035delA, c.181T>G, c. 3700_3704delGTAAA, c.68_69delAG)
test extended to include further mutations in BRCA1/2+CHEK2 + PALB genes (749delT(c.675delT), 185delAG (c.68_69delAG), $\mathrm{n}=7$ (10.3\%)
$5370>T$ (c.5251>T), 3819del5 (c.3700_3704delGTAAA), 3875del4 (c.3756_3759delGTCT), 8138del5 (c.7910_7914delCCTTT),
886 delGT (c.658_659deGT), 6174delT (c.5946delT), 5467insT (c.5239_5240insT), 4075delGT (c.3847_3848delGT)
CHEK2 gene (c.1100delC, del5395, c.444+1G>A, p.I157T)
PALB gene (c.172_175dellTTGT, C.509_510delGA)
next generation sequencing (NGS) of BRCA1/2 genes or multigene test

The majority of patients had genetic counseling (54-79.4\%). 6 patients $(10.3 \%)$ had a test for founder mutations in BRCA1 gene, 32 patients (47.1\%) had an extended test for further mutations in BRCA1/2 genes as well as in CHEK2 and PALB genes, 15 patients (22.1\%) had performed Next Generation Sequencing (NGS) of BRCA1/2 genes or a multigene test, and 16 patients (23.5\%) had no genetic test results found in their medical history (tab. II). In 17 patients (25\%), pathogenic mutations were found in BRCA1/2 genes.

No significant age differences were observed between BRCA1/2 gene mutation carriers and patients without the identified mutation (average 34.24 vs. 35.87; median 34 vs. 36). Patients who were carriers of mutations in BRCA1/2 genes were less likely ( $n=12 ; 70.6 \%$ ) to consult a reproductive medicine specialist than patients without known mutations ( $n=46$; $86.8 \%$ ), but no statistical significance was found. None of the BRCA1/2 mutation carriers opted for oocyte freezing.

Among the women with BRCA1/2 mutation, the GnRH analogues were used in 10 individuals (58.8\%), whereas among those without BRCA1/2 mutation, the $\mathrm{GnRH}$ analogues were included in 8 individuals (19\%) which was statistically significant (tab. III).

\section{Discussion}

Breast cancer in young women is rare, but the prognosis is poorer than in older women regardless of biological subtype. This includes a higher stage of the disease at the time of referral to the physician due to the glandular structure of the breast and associated diagnostic difficulties. More aggressive biological subtypes (triple-negative carcinoma, without expression of hormone receptors and without overexpression of the HER2 receptor or amplification of its gene, HER2-positive carcinoma showing overexpression of the HER2 receptor or amplification of its gene) and other molecular background are more com- mon in young women than in older breast cancer patients [4]. Young women are even over ten times more likely to be diagnosed with pathogenic mutations in genes that increase the risk of breast and ovarian cancer (BRCA1/2) than older women (2.5\% vs. 25\%) [5]. In the European Union, the average age at which the first child is born is 29.3 years, which means that often the diagnosis of breast cancer occurs among women who have not yet completed childbearing and are still planning offspring [6].

The principal treatment for breast cancer is surgery, but complementary treatments (chemotherapy, immunotherapy, hormone therapy, or radiotherapy) are also often used. Systemic treatment can significantly impair a woman's reproductive function, leading to temporary or irreversible infertility. The impairment of ovarian function depends on the patient's age, type of chemotherapy used, and the dose of drugs given. The commonly used perioperative chemotherapy involves anthracyclines and taxanes ( 4 x doxorubicin + cyclophosphamide, 12 x paclitaxel), and cytostatic drugs with an intermediate risk of causing permanent infertility in women. Infertility is always related with menopause induced changes like sexual distress. Chemotherapy-induced menopause symptoms can be more pronounced. Physiological menopause is a process, which lasts for many months, while iatrogenic menopause affects young patients experiencing a sudden decrease in estrogen level within a short time [7].

The authors of a paper published in July 2020 in the journal JAMA emphasize that despite ASCO recommendations, less than half of patients (44\%) with cancer of reproductive age - women aged 18 to 40, men aged 18 to 50 - were informed about the possibility of fertility disorders caused by cancer treatment and the possibility of consultation with a reproductive medicine specialist. This was more often the case for young, female patients, especially those suffering from breast cancer or hematological malignancies. Patients treated

Table III. Characteristics of patients according to genetic load

\begin{tabular}{|c|c|c|c|c|c|}
\hline $\begin{array}{l}\text { Pathogenic mutation in } \\
B R C A 1 / 2 \text { genes }\end{array}$ & $\begin{array}{c}\text { No. of } \\
\text { patients (\%) }\end{array}$ & $\begin{array}{l}\text { Average age } \\
\text { (years) }\end{array}$ & $\begin{array}{c}\text { Median age } \\
\text { (years) }\end{array}$ & Oocyte freezing & $\begin{array}{c}\text { Use of } \mathrm{GnRH}(\%) \\
\text { analogues }\end{array}$ \\
\hline current mutation & $n=17(25)$ & $n=34.24$ & $n=34$ & $n=0$ & $n=10(58.8)$ \\
\hline no mutation & $n=35(51.5)$ & $n=35.87$ & $n=36$ & $n=2(5.7)$ & $n=8(19 \%)$ \\
\hline no medical history & $n=16(23.5)$ & & & & \\
\hline
\end{tabular}


in academic centers or in places where fertility preservation procedures were reimbursed were better informed [8].

Also, according to the guidelines of Polish scientific societies, every patient with breast cancer of reproductive age should be informed about the possible complications of systemic treatment, including fertility disorders, which is confirmed by the results of our study [9]. The vast majority of patients qualified for the study, almost $80 \%$, had children. Twelve had a consultation with a reproductive medicine specialist, and 5 of them had already become mothers. Despite the patients' interest in fertility, only 2 patients decided to undergo oocyte freezing after oncological treatment and consultations with reproductive medicine specialists; this may be a result of the lack of reimbursement of fertility preservation procedures in Poland and in many countries and regions of the world.

The issue of informing patients about the problem of fertility and taking preventive measures is still unresolved. However, an evaluation of this problem was not the aim of this paper, and all patients were informed about the possibility of fertility disorders related to oncological treatment.

Young patients are found to carry pathogenic mutations in BRCA1/2 genes far more often than older patients. BRCA1/2 mutations increase the lifetime risk of breast cancer from $45 \%$ to $85 \%$ and ovarian cancer from $10 \%$ to $60 \%$. Effective measures to reduce the risk of breast and ovarian cancer in mutation carriers include a bilateral mastectomy and bilateral oophorectomy, which reduces the risk of cancer by $80-90 \%$. Mutation carriers who qualified for our study, and were aware of their situation, often took advantage of consultations with a reproductive medicine specialist and agreed to start $\mathrm{GnRH}$ analogues during systemic treatment. The prospect of needing cancer-reducing surgery in the future may be a factor prompting patients to implement fertility preservation procedures.

Ovarian stimulation, oocyte collection, oocyte freezing or fertilization and embryo freezing require postponement of the main oncological treatment by $3-4$ weeks. The effectiveness of presented methods reaches $30 \%$ and is similar to the effectiveness of in vitro fertilization in infertile couples without cancer history. In clinical practice, gonadotropin-releasing hormone analoges are also used during perioperative chemotherapy, the role of which is to inhibit ovarian function and thus reduce their susceptibility to cytostatic damage. However, the effectiveness of this method is limited [10].

The experience of our center highlights the importance of this problem and presents the decisions of patients who were offered the consultation of a reproductive medicine specialist and fertility preservation techniques. The awareness of fertility preservation by patients often results in reduced anxiety during therapy and improves cooperation between the patient and the oncologist.

\section{Conclusions}

Consultation with a reproductive medicine specialist should be a primary element of care for patients in their reproductive years who have been diagnosed with early breast cancer. The presence of pathogenic mutations in genes that increase the risk of breast and ovarian cancer (BRCA1/2) is an important factor in the decision to have offspring after treatment and before surgery with bilateral mastectomy and oophorectomy.

\section{Advantages and disadvantages of the study}

The study involved a homogeneous group of breast cancer patients. All patients were informed about the possible complications of cancer treatment, including impaired fertility. Decisions made by patients may differ from their actual beliefs and wishes due to the lack of reimbursement of fertility preservation procedures in Poland. The financial aspect may be the biggest factor for breast cancer patients to take action.

Conflict of interest: none declared

\section{Mikołaj Bartoszkiewicz \\ Poznan University of Medical Sciences \\ Department of Immunobiology \\ ul. Rokietnicka 8 \\ 60-806 Poznań, Poland \\ e-mail:m.bartoszkiewicz@ump.edu.pl}

Received: 22 Mar 2021

Accepted: 15 Jun 2021

\section{References}

1. Assi HA, Khoury KE, Dbouk $\mathrm{H}$, et al. Epidemiology and prognosis of breast cancer in young women. JThorac Dis. 2013; 5 Suppl 1: S2-S8, doi: 10.3978/j.issn.2072-1439.2013.05.24, indexed in Pubmed: 23819024.

2. Oktay K, Harvey BE, Loren AW, et al. Fertility Preservation in Patients With Cancer: ASCO Clinical Practice Guideline Update. J Clin Oncol. 2018; 36(19): 1994-2001, doi: 10.1200/JCO.2018.78.1914, indexed in Pubmed: 29620997.

3. Narod SA. BRCA mutations in the management of breast cancer: the state of the art. Nat Rev Clin Oncol. 2010; 7(12): 702-707, doi: 10.1038/ nrclinonc.2010.166, indexed in Pubmed: 20956982.

4. Erić I, Petek Erić A, Kristek J, et al. BREAST CANCER IN YOUNG WOMEN: PATHOLOGIC AND IMMUNOHISTOCHEMICAL FEATURES. Acta Clin Croat. 2018; 57(3): 497-502, doi: 10.20471/acc.2018.57.03.13, indexed in Pubmed: 31168183.

5. Abdel-Razeq H, Al-Omari A, Zahran F, et al. Germline BRCA1/BRCA2 mutations among high risk breast cancer patients in Jordan. BMC Cancer. 2018; 18(1): 152, doi: 10.1186/s12885-018-4079-1, indexed in Pubmed: 29409476.

6. EUROSTAT. Women are having their first child at an older age. https:// ec.europa.eu/eurostat/web/products-eurostat-news/-/DDN-202005152 (11.02.2021).

7. Lachowicz M, Kufel-Grabowska J, Bartoszkiewicz M, et al. Sexual well being of breast cancer patients. Nowotwory. Journal of Oncology. 2021; 71(4): 232-237, doi: 10.5603/njo.a2021.0038.

8. Patel P, Kohn TP, Cohen J, et al. Evaluation of Reported Fertility Preservation Counseling Before Chemotherapy Using the Quality Oncology Practice Initiative Survey. JAMA Netw Open. 2020; 3(7): e2010806, doi: 10.1001/jamanetworkopen.2020.10806, indexed in Pubmed: 32678451.

9. Jassem J, Krzakowski M, Bobek-Billewicz B, et al. Rak piersi. Onkol Prakt Klin Edu. 2020; 6(5): 297-352.

10. Ter Welle-Butalid ME, Vriens IJ, Derhaag JG, et al. Counseling young women with early breast cancer on fertility preservation. J Assist Reprod Genet. 2019; 36(12): 2593-2604, doi: 10.1007/s10815-019-01615-6, indexed in Pubmed: 31760547. 